\title{
Indicadores de Gestión del Conocimiento en LA FACULTAD dE INGENIERÍA INDUSTRIAL
}

\author{
Recepción: Noviembre de 2004 / Aceptación: Diciembre 2004
}

\author{
(1) Jorge Inche Mitma \\ (2) Alfonso Chung Pinzás
}

\section{RESUMEN}

El artículo trata sobre el análisis de los indicadores de Gestión del Conocimiento en la Facultad de Ingeniería Industrial, para una propuesta futura de un Modelo de Gestión del Conocimiento y un Tablero de Mando Integral, para el control del capital más importante en una organización, el capital intelectual.

Palabras Claves: Gestión del conocimiento. Tablero de mando integral. Capital intelectual.

KNOWLEDGe Management Indicators at the Facuity of Industrial Engineering ABSTRACT

This article deals about the analysis of knowledge management indicators at the Faculty of Industrial Engineering, for the future proposal of a Knowledge Management Model and an Integral Control Board, for controlling the most important capital in an organization, namely intellectual capital.

Key Words: Knowledge management. Integral control board. Intellectual capital.

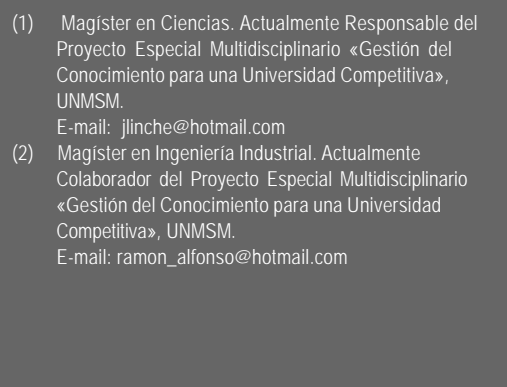

\section{INTRODUCCIÓN}

Uno de los aspectos más importantes dentro de cualquier organización es el capital intelectual que esta posee. Sin embargo, en nuestra realidad, este aspecto es muchas veces dejado de lado por rubros "tangibles" como por ejemplo, la adquisición de maquinarias y equipos, ingresos monetarios, etc.

En una universidad, ya sea nacional o privada, conocer este capital es un aspecto vital ya que representa su razón de existir, es decir la investigación y la generación de conocimiento, por eso es necesario medir y comparar el capital intelectual que ésta posee. Es así que en la Facultad de Ingeniería Industrial de la UNMSM se está llevando a cabo este proyecto mediante un grupo multidisciplinario de investigadores de la Facultades de: Economía; Ingeniería de Sistemas e Informática; Ingeniería Industrial; Ingeniería Electrónica; Ingeniería Geológica, Minera, Metalúrgica y Geográfica; Ciencias Administrativas.

En este contexto, el problema se formula en la siguiente interrogante: ¿Cómo medir y controlar el capital intelectual en una universidad nacional?. El estudio pretende gestionar los indicadores de capital intelectual, para esto será necesario buscar estándares en pares gemelos de tal forma que puedan servir de punto de referencia para las comparaciones correspondientes.

Las limitaciones están dadas por la disponibilidad de información ya que es necesario información de al menos 5 años atrás para realizar proyecciones estadísticas. Esta limitación permitió establecer una etapa inicial de análisis de indicadores piloto en la Facultad de Ingeniería Industrial.

\section{ANÁLISIS DE LOS INDICADORES DE GESTIÓN DEL CONOCIMIENTO}

\section{Indicadores de Primer Nivel}

Se conocen como de primer nivel a aquellos indicadores que son lineales, es decir, no son producto de ninguna operación; por ejemplo:

- No. de Convenios Internacionales $=5$

- No. de Tesis de doctorado $=2$

Indicadores de Segundo Nivel Los indicadores de segundo nivel son aquellos que se establecen como un cociente, por ejemplo: 
- No. de Convenios Internacionales / No. de Investigadores $=0,23$

- No. de Tesis de Doctorado / No. de Doctorandos = 0,14

Indicadores de Tercer Nivel

Aquellos que se expresan en porcentajes, por ejemplo:

- Porcentaje de Financiamiento de Proyectos del Tesoro Público = 5\%

\section{FASES DEL PROYECTO}

El proyecto se divide en las siguientes fases:

1. Formación del equipo de trabajo.

2. Formulación de indicadores decapital intelectual.

3. Verificación de los indicadores de capital intelectual.

4. Formulación del tablero de mando integral.

5. Presentación del modelo de gestión de conocimiento.

6. Verificación del modelo.

A continuación se explica cada fase del proyecto :

\section{Formación del Equipo de Trabajo}

La formación debe ser multidisciplinaria, procurando la presencia de expositores sobre gestión del conocimiento; se recomienda la presencia de investigadores con conocimientos en software y estadística.

\section{Formulación de Indicadores de Capital In- telectual}

La formulación de indicadores pasa por la revisión de experiencias anteriores, evaluando los resultados, discutiendo la pertinencia de dichos indicadores hasta lograr el consenso en el tema.

\section{Verificación de los Indicadores de Capital Intelectual}

La verificación implica el llenado por años de los indicadores, para esto el equipo de trabajo deberá establecer formatos para entrevistas en las diversas oficinas de las Facultades, en especial a los Institutos de Investigación y, en mucho casos, los datos tendrán que ser estructurados ya que se encuentrandispersos.

\section{Formulación del Tablero de Mando Integral}

Para la formulación del tablero deberá investigarse los pares gemelos contra quienes comparar, parale- lamente se deberá elaborar el tablero de mando y al mismo tiempo su programación en un software.

\section{Presentación del Modelo de Gestión del Conocimiento}

El modelo de Gestión del Conocimiento incorpora los procedimientos para la valorización del capital intelectual en términos monetarios, los cuales se controlan a través del Tablero de Mando Integral.

\section{Verificación del Modelo}

La validación del modelo está referida al análisis comparativo con otras Facultades de Ingeniería entre Universidades, mediante ensayos estadísticos.

\section{CAPITAL INTELECTUAL}

\section{Capital Humano}

"Conocimiento de las personas (capacidad y compromiso)».

Es el valor de lo que los individuos pueden producir, tanto individual como, sobre todo, colectivamente. Tiene que ver con las competencias (conocimientos, habilidades y cualidades profesionales), con la capacidad de innovar y mejorar, y con el compromiso y la motivación (dedicación y calidad en la actuación). En definitiva, conocimiento aplicado muy relacionado con el compromiso.

El modelo de Gestión del Conocimiento con relación al capital humano se centra en los siguientes aspectos:

1. Las competencias, que se materializan en:

a. Gestión de la formación que supone contemplar la cantidad de formación, su calidad, la aplicabilidad y el acceso a la misma.

b. Gestión para la definición y difusión de competencias o valores necesarios para alinearse con la estrategia y el logro de los objetivos.

2. La capacidad de innovar y mejorar, que se materializa en:

a. Gestión de la diversidad.

b. Gestión de la colaboración.

c. Gestión de la iniciativa y de la creatividad.

d. Gestión del aprendizaje.

e. Gestión del cambio.

3. El compromiso y la motivación, que se materializan en:

a. Gestión participativa: interiorización y compromiso con el proyecto.

b. Gestión del conocimiento, la motivación y la 
compensación.

c. Gestión de la comunicación interna: canales, mensajes, etc.

d. Gestión del clima de trabajo.

e. Gestión del cumplimiento.

No cabe duda que en la Universidad se da con singularidad la formación de capital humano, ya que su propia esencia se sustenta en una concepción basada en el estudio, la investigación, la creatividad, la crítica, la generación de nuevas ideas científicas y tecnológicas, el avance en el conocimiento, la transmisión y comunicación mediante la actividad docente, para colegas y alumnos, que desemboca en el aprendizaje y conllevan la propia formación.

\section{Capital Estructural}

"Conocimiento de la organización (conocimiento, tecnología y cultura)».

Es el valor del conocimiento clave sistematizado, empaquetado, difundido y accesible, conformado por los procesos, medios, infraestructuras, tecnología, sistemas, controles, etc., que posibilitan la creación de valor.

Por eso algunos (Edvinsson y Malone, 1999) lo separan en capital innovación (identificado como la renovación y los resultados de la innovación en forma de derechos comerciales protegidos, propiedad intelectual y otros activos intangibles, así como los talentos usados para crear y llevar rápidamente al mercado nuevos productos y servicios) y capital proceso (identificado como los procesos de trabajo, técnicas y programas para empleados que aumentan y fortalecen la eficiencia de producción o la prestación deservicios).

El modelo de Gestión del Conocimiento con relación al capital estructural se centra en los siguientes aspectos:

1. Las infraestructuras de relación con el cliente, que se materializan en:

a. Despliegue de redes comerciales y canales de autoservicio y complementarios.

b. Localización y capacidad de atención de los canales.

2. Las tecnologías y la calidad de los procesos, que se materializan en:

a. Despliegue tecnológico: en cantidad, modernidad, capacidad adaptación al usuario.

b. Tecnologías de producto.

c. Gestión de la arquitectura de la información

d. Certificaciones ISO, auditorias y resultados.
3. La organización y los sistemas de dirección y gestión, que se materializan en:

a. Actualización de las estructuras organizativas: funciones, organigramas, etc.

b. Sistemas de información de gestión

c. Resultados de la función auditoria.

En el ámbito de la Universidad son funciones básicas, a las que se deben dedicar grandes esfuerzos de desarrollo y mejora, los procesos docentes e investigadores: matricula de alumnos, organización de planes docentes, expedición de títulos, selección de profesorado, asignación de becas, reglamentos de Departamentos y Centros, reglamento de alumnos, asignación de proyectos de investigación, tramitación de contratos de investigación, gestión presupuestaria, gestión de sistemas, servicio de informática y centro de cálculo, gestión de convenios, proceso de evaluación de la calidad de las distintas áreas, aprovechamiento de nuevas tecnologías de innovación educativa y campus virtual, procesos de aprendizaje, etc.

\section{Capital Relacional}

"Conocimiento del entorno aplicado o forma de relación de la organización con el exterior (base y valor de marca)».

Es el valor de la base de clientes, de la capacidad de mantener la relación con ellos y de su potencial, y el valor referido a otros agentes del entorno como los proveedores, las autoridades, etc.

Algunos lo separan en capital comercial (se centra en las relaciones con clientes y proveedores y en el conocimiento del grado de satisfacción de éstos) y capital comunicación (relacionado con actividades de comunicación con el exterior dentro de las actividades demarketing).

El modelo de gestión con relación al capital relacional se centra en los siguientes aspectos :

1. Lealtad y vinculación, que se materializan en:

a. Gestión de la calidad: interna y perciba por el cliente.

b. Gestión de la relación con los clientes.

c. Cuotas de mercado y cuotas de clientes.

d. Gestión de la marca.

2. Intensidad, colaboración y conectividad, que se materializan en:

a. Gestión de canales: individual e integradamente.

b. Gestión de convenios con clientes institucionales.

c. Gestión de la imagen corporativa.

d. Gestión de relaciones con la sociedad.

e. Gestión de alianzas estratégicas. 
Cuadro 1. Indicadores de primer nivel de gestión del capital intelectual en la Facultad de Ingeniería Industrial, UNMSM

\begin{tabular}{|c|c|}
\hline $\begin{array}{l}\text { INDICADORES DE PRIMER NIVEL } \\
\text { (Por Facultad-Año 2004) }\end{array}$ & RESULTADO \\
\hline \multicolumn{2}{|l|}{ CAPITAL HUMANO } \\
\hline No. de Doctores en la Plana Docente & 0 \\
\hline No. de Magísteres en la Plana Docente & 11 \\
\hline No. de Titulados en la Plana Docente & 50 \\
\hline No. de Docentes Capacitados & 4 \\
\hline No. Total de Docentes & 50 \\
\hline No. de Docentes con Labor Administrativa & 12 \\
\hline No. de Grupos de Investiqación Activos & 2 \\
\hline No. de Egresados de Pregrado & 106 \\
\hline No. de Eqresados de Maestría & 7 \\
\hline No. de Egresados de Doctorado & 0 \\
\hline No. de Docentes Investigadores con grado de Doctor[1] & 0 \\
\hline No. de Docentes Investigadores con grado de Magíster[2] & 7 \\
\hline No. Total de Docentes Investigadores & 47 \\
\hline Monto Destinado a los Docentes Investigadores (S/.) & 32000 \\
\hline Incentivo Promedio a los Docentes Investigadores (S/.) & 164 \\
\hline No. de Consultores con más de 5 Trabajos para Instituciones Públicas y Privadas & 6 \\
\hline No. de Proyectos de Investigación & 4 \\
\hline \multicolumn{2}{|l|}{ CAPITAL ESTRUCTURAL } \\
\hline No. de Libros Publicados con Registro ISBN & 2 \\
\hline No. de Libros con Antigüedad no mayor de 5 años en la Biblioteca & 1423 \\
\hline Total de Libros en la Biblioteca & 6141 \\
\hline No. de Programas de Doctorado & 1 \\
\hline No. de Programas de Maestrías & 2 \\
\hline No. de Programas de Diplomados & 0 \\
\hline No. de Proyectos de Investigación con Organismos No Gubernamentales & 0 \\
\hline No. de Proyectos de Investigación con Entidades Públicas & 0 \\
\hline No. de Proyectos de Investigación con el Sector Empresarial & 0 \\
\hline Número de Laboratorios & 4 \\
\hline No. de Softwares Usados en los Cursos de Pregrado & 20 \\
\hline No. de Softwares Usados en los Cursos de Postarado & 4 \\
\hline No. de Computadoras de Última Generación & 75 \\
\hline No. de Libros Generados de la Investiqación[3] & 1 \\
\hline No. de Artículos en Revistas Indexadas & 12 \\
\hline No. de Artículos en Revistas & 24 \\
\hline No. de Patentes & 0 \\
\hline No. de Modelos o Prototipos & 0 \\
\hline No. de Tesis Doctorales & 0 \\
\hline No. de Tesis de Maestría & 2 \\
\hline No. de Tesis de Pregrado & 22 \\
\hline No. de Líneas de Investigación Activas & 3 \\
\hline No. de Tesis de Pregrado Financiadas & 5 \\
\hline No. de Tesis de Maestría Financiadas & 0 \\
\hline No. de Tesis Doctorales Financiadas & 0 \\
\hline \multicolumn{2}{|l|}{ CAPITAL RELACIONAL } \\
\hline No. de Convenios Internacionales & 1 \\
\hline No. de Convenios Nacionales & 1 \\
\hline No. de Congresos Nacionales Organizados & 0 \\
\hline No. de Congresos Internacionales Organizados & 0 \\
\hline No. de Seminario y Otros Eventos Internacionales Organizados & 0 \\
\hline No. de Ponencias en Congresos Internacionales & 2 \\
\hline No. de Ponencias en Congresos Nacionales & 0 \\
\hline No. de Premios Concedidos & 0 \\
\hline No. de Premios Recibidos & 2 \\
\hline No. de Estancias de Investigación Nacionales & 0 \\
\hline No. de Estancias de Investiqación Internacionales & 0 \\
\hline No. de Docentes Miembros de Sociedades Científicas Internacionales & 2 \\
\hline No. de Docentes Miembros de Sociedades Científicas Nacionales & 0 \\
\hline
\end{tabular}

Fuente: Facultad de Ingeniería Industrial, UNMSM

[1] OCCA (2005), Indicadores de Gestión para la UNMSM. En: http://www.unmsm.edu.pe/Noticias2005/marzo/d2/veramp.php?val=1

[2] Idem

[3] Idem 
En la Universidad se tiene que potenciar todos los aspectos relacionados con la oficina de orientación de alumnos, el servicio de inserción al mundo laboral, la asociación de antiguos alumnos, el servicio de prensa-revistas, folletos, CD y página web, la formación continua, el servicios de redes, los servicios de prácticas en empresas o vinculación, la oficina de relaciones laborales, el servicio de residencias o colegios mayores, los servicios deportivos y culturales, los servicios docentes y de biblioteca, el servicio de protocolo, la oficina del defensor del universitario, la oficina de relaciones interuniversitarias, etc.

\section{AVANCES DEL PROYECTO}

El equipo de investigación está desarrollando el proyecto en su fase piloto en la Facultad de Ingeniería Industrial de la UNMSM y se encuentra en la fase verificación de los indicadores de capital intelectual.

En el Cuadro 1 se presentan los indicadores de primer nivel para la Facultad de Ingeniería Industrial.

Cabe mencionar las dificultades en la recolección de información de campo, como se mencionó anteriormente, algunos datos no se tenían estructurados así que se tuvo que clasificarlos para luego recolectarlos.

Los resultados dan una idea general de la situación de la Facultad, más aún si se piensa en una futura comparación de resultados.

\section{CONCLUSIONES Y RECOMENDACIONES}

La Gestión del Conocimiento no sólo debe implicar la formulación de indicadores, sino que estos deben proporcionar una información sobre su estado; esto se logra mediante la aplicación de un Tablero de Mando Integral.
El capital intelectual es el patrimonio más importante de una organización, por lo tanto su medición es un índice vital para su mejor desarrollo.

La ubicación de pares gemelos como estándares de comparación, es un proceso bastante delicado ya que se debe hacer la selección teniendo en cuenta su compatibilidad con la situación actual de la universidad a comparar.

Una de las mayores dificultades que se presenta en este tipo de estudio es la falta de datos para los indicadores, mas aún si se desea tener una evolución de 5 años atrás; por ello se recomienda solicitar la información con anterioridad a la fecha esperada de recolección.

\section{BIBLIOGRAFÍA}

1. Bueno, Eduardo. (2002). La Gestión del Conocimiento Científico y Técnico en la Comunidad de Madrid. Un Proyecto en Marcha. Universidad Autónoma de Madrid. Madrid, España.

2. Bueno, Eduardo. (2003). Gestión del Conocimiento en Universidades y organismos Públicos de Investigación. Ediciones de la Dirección General de Investigación - Consejería de Educación. Madrid, España.

3. Zamorano, Héctor. (2003). Modelos de Simulación para la Gestión del Conocimiento. En: www.citynet.com.ar/estudio. Argentina.

4. OCCA (2005). Indicadores de Gestión para la UNMSM. En: http://www.unmsm.edu.pe/Noticias2005/marzo/d2/veramp.php?val=1

5. Madrid + D. (2002). Tablas de Indicadores de Capital Intelectual. En: http://www.madrimasd.org/ informacionidi/indicadores/intelectual/indicadores/ default.asp 